\title{
The psychological and EEG effects of 5-HTTLPR gene polymorphism among people from different ethnic groups in Siberia
}

\author{
A.N. Savostyanov ${ }^{1,2,3 *}$, D.V. Bazovkina ${ }^{2}$, A.N. Saprygin ${ }^{1}$, S.S. Tamozhnikov ${ }^{1}$, \\ T.A. Ausheeva ${ }^{3}$, A.V. Bocharov ${ }^{1,3}$, U.N. Kavai-ool ${ }^{4}$, A.G. Karpova ${ }^{5}$, G.G. Knyazev ${ }^{1}$ \\ ${ }^{1}$ Institute of Physiology and Basic Medicine, Novosibirsk, Russia \\ ${ }^{2}$ Institute of Cytology and Genetics of SB RAS, Novosibirsk, Russia \\ ${ }^{3}$ Novosibirsk State University, Novosibirsk, Russia \\ ${ }^{4}$ Tuvan State University, Kyzyl, Russia \\ ${ }^{5}$ North-Eastern Federal University, Yakutsk, Russia \\ *e-mail: Alexander.Savostyanov@gmail.com
}

Key words: 5-HTTLPR, EEG, Ethnic differences

Motivation and Aim: Serotonin transporter is one of the most widely investigated genetic markers of individual variation in serotonergic function. The promoter region of the serotonin transporter gene (5-HTTLPR) contains long (L) and short (S) variants. The 5-HTTLPR allele polymorphism of serotonin transporter (5-HTT) is associated with inclination to anxietyrelated psychopathology, including depression. However, the effect of 5-HTTLPR to the risk of depression and anxiety disorder is highly variable in different ethnic groups. According to the most of reports, an S- allele has been found to increase the risk of mental health problems in the Caucasoids, but also the same allele has an opposite effect or no effect on the different Mongoloid groups. This study comparatively investigated the effects of 5-HTTLPR polymorphism on psychological measures, EEG current source density, connectivity, and topological properties of resting state networks, and the ERSP indexes under recognition of emotional-related stimuli in the groups of Caucasoid Russians (210 persons) and Siberian Mongoloids - Tuvinians, Yakuts and Evenks (183 persons).

Methods: The trait anxiety level, the neuroticism scores and the emotional intelligence scores were measured in all participants by means of different self-report inventories. EEG was recorded in the Russians by means of the 128-channel and in Mongoloids by means of 64-channel Brain Products amplifier, Germany. EEG was recorded during the resting state, during execution of experimental tasks, which were related with recognition of facial and speech emotionality.

Results: SS homozygotes showed higher scores of anxiety and neuroticism and lower scores of the emotional intelligence in comparison with the L-allele carriers among all ethnic groups. The ERSP indexes of theta synchronization in the people with L-genotype showed the amplitude difference for recognition of stimuli with different emotional modalities, whereas there were not detected such differences in the SS homozygotes for all ethnic groups. As compared to L-carriers, S homozygotes from the Caucasoid group showed lower current source density and connectivity in most frequency bands in areas overlapping with the default mode and emotion regulation regions. However, the opposite effect of the $\mathrm{S}$ allele was revealed in the Mongoloid group, i.e. the Mongoloid S homozygotes showed higher current source density and connectivity in the same brain areas and frequency bands in comparison with the Mongoloid L-carriers. In addition, the associations between restingstate EEG and these psychological measures were different among ethnic groups.

Conclusion: The effect of 5-HTTLPR polymorphism on EEG and on association of restingstate EEG networks with psychometric measures looks unstable and depends on many additional climatic and cultural parameters.

Acknowledgments: The study was supported by the Russian Science Foundation (RSF) under Grant No. 17-18-01019. Data collection in the Yakutian group was supported by the grant 18-415-140021 p_a of the Russian Foundation of Basic Research (RFBR). 